\title{
Comparison of Test-retest Measurement Reliability of Iliopsoas Strength between Break and Make Test in Subjects with Lumbar Extension Syndrome
}

\author{
In-cheol Jeon, PT, Ph.D \\ Department of Physical Therapy, Hoseo University, Asan, South Korea
}

Background The strength of iliopsoas muscle is frequently measured to evaluate the performance of hip flexion in the clinical setting. However, little research into the test- retest reliability of iliopsoas muscle strength in subjects with lumbar extension syndrome (LES) in supine position has been reported.

Purpose The purpose was to compare the test- retest reliability of iliopsoas strength measurement in supine position with break and make test in subjects with LES. The iliopsoas muscle is very important in the hip joint structures. The reliable measurement is essential to evaluate the performance of hip flexion.

Study design Reliability study.

Methods Twenty subjects were enrolled in this study. The strength of iliopsoas muscle was measured in supine position, with using a smart KEMA tensiometer system for make test, using a hand held dynamometer for break test. The intra class correlation coefficients (ICC 3, 1) were used to determine test-retest reliability of iliopsoas muscle strength.

Results Test-retest reliability of iliopsoas muscle strength measurements in make test was significantly greater compared with break test $(p<0.05)$. The value of ICC with make test was greater than make test ( 0.86 for make test, 0.71 for break test). Additionally, the coefficients of variation ( $4.5 \%$ for make test, $18.3 \%$ for break test) was better in the supine position with make test.

Conclusions The measurement of iliopsoas muscle strength in make test can be more reliable JMST

\section{CONTACT}

\section{This is an Open-Access article distributed under the terms of the Creative Commons Att- ribution Non-Commercial Li- cense (http://creativecommons org/licenses/by-nc/4.0) which permits unrestricted non-co- mmercial use, distribution, and reproduction in any me- dium, provided the origina} work is properly cited. than break test in subjects with LES in supine position. The make test in supine position can be recommended for reliable measurement of iliopsoas muscle in subjects with LES.

Key words Break test; Iliopsoas; Lumbar extension syndrome; Make test; Strength measurement.

\section{INTRODUCTION}

The iliopsoas muscle is very important in the stabilization of hip joint structure. ${ }^{1}$ The iliopsoas muscle was in function as a hip flexor and as a dynamic stabilizer of hip joint during walking ${ }^{2}$ and active leg raising. ${ }^{3}$ In addition, the iliopsoas muscle can contribute to maintaining the continuous axis of femur head in hip joint during hip extension and external rotation. ${ }^{1,2}$ The insufficient muscle strength of the iliopsoas muscle can result in the frequent and excessive compensations of femur head during hip extension and external rotation in supine and standing position causing the femur anterior gliding syndrome. ${ }^{4}$ Therefore, the precise measurement of iliopsoas muscle strength is one of the primary important methods in hip joint rehabilitation. ${ }^{1}$

A tight iliopsoas muscle and rectus femoris muscle can cause the influence on low back pain (LBP) and result in alterations in the movements strategy. ${ }^{4}$ Among the five 
subcategories of lumbar spine with pain and abnormal patterns identified by Sharmann ${ }^{1}$, lumbar extension syndrome (LES) is one of the most common syndrome in the clinical setting. The LES was caused by muscle imbalance between the abdominal muscles and hip flexor muscles such as iliopsoas and rectus femoris. The tight hip flexors and insufficient performance of abdominal muscles can increase the anterior shearing force of the lumbar spines causing hyper-lordosis or facet joint syndrome in standing and supine position. ${ }^{1}$ The optimal length of hip flexors and the sufficient performance of the abdominal muscles are required to maintain the normal posture of lordotic curve in the lumbar spines in standing and supine position. ${ }^{1}$ Therefore, Excessive changes in the abdominal muscle performance such as Transverse abdominis and internal oblique muscle and the hip flexor length may cause functional limitations and movement impairments during hip flexion in standing and supine position. ${ }^{1,2}$

A hand held dynamometer (HHD) is a conventional tool for clinical measurement of muscle strength. ${ }^{5,6}$ The HHD is effective for an quick measurement of objective values in clinic and experimental environment. However, Schwartz ${ }^{7}$ reported that the use of HHD is less sensitive for upper extremity strength graded more than 4 . In addition, the correlation coefficients for lower-extremity testing between examiners were much lower than those for upper extremity strength measurement. The inter-rater reliability for hip flexor was 0.74 , whereas for the measurement of shoulder flexor was 0.94 in healthy subjects. ${ }^{8}$ An inter-rater reliability contributed to a values of association between repeated tests. Some previous studies reported that HHD was not reliable for the measurement of the lower-extremity. ${ }^{6}$ On the other hand, Hip flexion in supine position can be performed to be measured by HHD with using a long lever arm. 5,9

Hip flexion in supine position can be the functional movement for testing the strength and performance of the iliopsoas. As a precise measurement of the iliopsoas is an important component in successful rehabilitation of subjects with LES, the precise strength is required to be measrued in clinical and research settings. The HHD was used to perform break test with manual resistance during hip flexion in supine position. The smart KEMA tensiometer system was used to perform make test with performing hip flexion in non-elastic band. The optimal body posture for the measurement of iliopsoas strength was investigated as a supine position in subjects with LES. ${ }^{1}$ no prior study has compared the test-retest measurement reliability of the iliopsoas strength between break and make tests in subjects with LES. The purpose of the present study was to compare the test-retest reliability of the strength measurement of the iliopsoas between break and make test in supine position in subjects with LES. We hypothesized that the reliability would be better in supine position with make test than break test.

\section{METHODS}

\section{Participants}

G*Power software was employed (ver. 3.1.2; Franz Faul, University of Kiel, Kiel, Germany) in a pilot study of ten participants. The calculation of the sample size was performed with a power (0.80), an alpha level (0.05), and an effect size (1.27). This result reported that the sample size was required at least fifteen participants. Twenty subjects with LES aged 20-30 years were participated in this study (Table 1). Inclusion criteria included: (1) normal hip joint range of motion, (2) no dysfunctions of musculoskeletal and neurological region, (3) no surgery of history of hip joint, (4) a positive sign of Ely's test and modified Thomas test. Exclusion criteria included: (1) specific LBP with radiation symptom, (2) hip or knee joint surgery, and (3) hip and knee joint contractures. To classify LES, we used the evaluation of methodology guided by Sahrmann. ${ }^{1}$ The classification methods consist of the alignment and movement tests. And the movement tests consist of primary (pain provoking tests) and secondary (pain reducing tests) tests. ${ }^{1}$ Several tests were performed to classify the syndrome of LES. The detailed methods of several tests for diagnosis of LES have been described in the previous stydy. ${ }^{10}$ The experimental protocols were explained in detail to subjects, and all subjects provided written informed consent with the ethical principles of the Declaration of Helsinki.

\section{Procedures}

The maximal strength of the iliopsoas was measured in

Table 1. General characteristics of the subjects $\quad(\mathrm{N}=20)$

\begin{tabular}{lc}
\hline & Mean \pm SD \\
\hline Age (years) & $26.1 \pm 5.2$ \\
Height (cm) & $170.2 \pm 5.1$ \\
Body mass (kg) & $65.1 \pm 4.2$ \\
Visual analogue scale (mm) & $61.5 \pm 5.2$ \\
Duration of low back pain (years) & $1.5 \pm 0.5$ \\
Modified Oswestry Disability & $14.5 \pm 7.4$ \\
Index (\%) & 14 males, 6 females \\
\hline Gender & \\
\hline
\end{tabular}


supine position using the dominant leg. The strength measurement of the dominant side was performed as a tested side. To measure the strength of the iliopsoas muscle, the break test and the make test were performed in supine position. For the break test, a hand-held dynamometer (HHD; Lafayette Instrument Company, Lafayette, IN, USA) was employed (Figure 1). For the make test, maximal strength of the iliopsoas muscle was measured with a tensiometer using a non-elastic band (Smart KEMA tensiometer sensor; Factorial Holdings Co., Ltd., Seoul, Korea) (Figure 2).

The specific resistance region for the iliopsoas muscle was placed at the region $5 \mathrm{~cm}$ below the knee joint in supine position. ${ }^{11}$ And a straight line on the skin at the same region was marked to eliminate the regional difference. ${ }^{2}$ The measurements were performed on the same day to measure the test-retest reliability. The tests order between break and make test were randomized. Prior to the experimental procedure, the subjects were familiarized with the make and break tests to eliminate measurement errors. A Smart
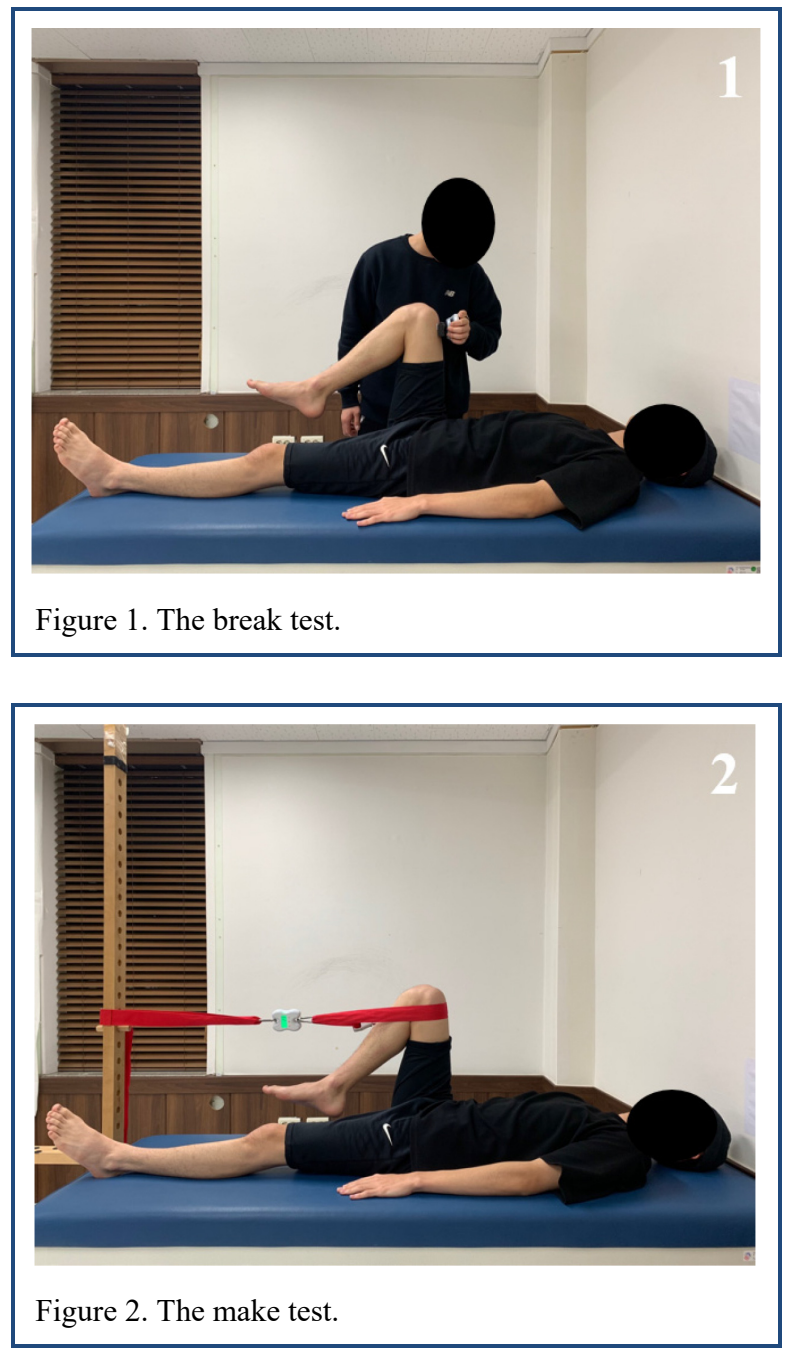

KEMA tensiometer using a non-elastic band was employed to measure the iliopsoas strength for make test. The HHD was used to measure the iliopsoas strength for break test. For the measurements, the subjects were asked to perform knee and hip flexion at 90 degree in tested side during the strength measurements. ${ }^{10}$ The hip and knee joint in nontested side were fully extended to maintain the supine position. The duration of contractions for strength measurements was maintained for approximately 5 seconds to measure the iliopsoas strength. The maximal strength provided by the tensiometer and by the HHD was retained in kilograms. Each task was performed with 3 times and all values of the measurement were averaged.

\section{Statistical analysis}

Statistical analysis was conducted using SPSS program (ver. 21.0; SPSS, Inc., Chicago, IL, USA). ICC with 95\% confidence intervals (CIs) were calculated. The $\operatorname{ICC}(3,1)$ model was used to calculate intra-rater reliability of averaged strength. In the interpretation, the ICC value $(>0.75)$ was used to indicate "excellent," $(0.40-0.75)$ was "fair to good," and (0.00-0.40) was "poor". ${ }^{12,13}$

\section{RESULTS}

The test-retest ICC of the strength measurement of iliopsoas for the make test $(0.86$; 0.81-0.89 with 95\% confidence interval; $19.37 \pm 3.64(\mathrm{~kg})$ with averaged strength) was greater than that for the break test $(0.71 ; 0.65-0.76$ with $95 \%$ confidence interval; $17.19 \pm 6.72(\mathrm{~kg})$ with averaged strength). The coefficients of variations were $4.5 \%$ for make test, $18.3 \%$ for break test.

\section{DISCUSSION}

The purpose of this study was to compare the test-retest measurement reliability of the iliopsoas strength in supine position in subjects with LES using make and break tests. This research is the study to compare the test-retest reliability of break and make test of the strength measurement of the iliopsoas in supine position in subjects with LES. The result of this study reported that the test-retest ICC of the make test was greater than that of the break test in subjects with LES. The break test with an HHD is the conventional way in clinical setting to measure the muscle strength, the make test with a tensiometer is less common. These tests were used to measure iliopsoas muscle strength based on differences in resistance between break test (eccentric contractions) and make test (isometric contrac- 
tions).

There was some explanation to explain these findings. First, The strength of the iliopsoas with break test was smaller than make test because of length-tension relationship of insufficient performance of the abdominal muscles. ${ }^{4,7,8}$ The end position of break test to measure the iliopsoas strength in supine position may be different from the initial position which was in knee and hip flexion at 90 degree because of insufficient co-contraction of the abdominal muscles. $^{2,3}$ Although the direct comparison of findings between the previous and this study was not possible, these results may indicate that the performance of the hip flexor muscles can be related to abdominal muscle performance and neutral pelvic posture in supine position especially in subjects with LES. $^{3}$ Second, the measurement with make test by assessing isometric contractions may have contributed to its greater test- retest reliability compared to those with the break test. A Smart KEMA tensiometer using a nonelastic band was used to maintain a consistent hip flexion angle in supine position. Although fatigue of the iliopsoas muscle may be occurred, the non-elastic band used for the make test may have help to maintain the consistent flexion angle ${ }^{14}$ because the end position for make test might be maintained within the acceptable range of the non-elastic band. In contrast, the break test with a HHD may result in subtle compensations in the hip flexion angle in supine position depending on the iliopsoas and the abdominal muscle performance, especially in subjects with LES. Third, the potential reason why the reliability of make test was greater than the break test in this study was that possible adaptation with constant task position for the examiner and subject. $^{2,3}$ The measurement for make test was easily accustomed to the subject's hip flexion angle in supine position and the examiner. However, the measurement for break test was easily different to the subject's test position including hip flexion angle affected by insufficient performance of the abdominal muscle in subjects with LES. ${ }^{15}$ So, the results of this study reported that the various elements affecting the test-retest reliability should be set to minimize possible measurement errors. ${ }^{16}$

The measurement device used for the make test have advantages, such as cost-effectiveness and easy application. These characters may make it more accessible in the clinical setting. Therefore, the make test for the measurement of the iliopsoas muscle strength in supine position can be recommended for more reliable method in subjects with LES.

The current study had several limitations. First, the EMG was not used to investigate the abdominal and iliopsoas muscle. Further study is required to investigate the EMG of the abdominal and iliopsoas muscle during hip flexion in supine position. Second, variations in the severity of LES were not considered. Further study is required to investigate the reliability of the strength measurement according to the severity of LES. Third, the results cannot be generalized to elderly subjects. Further study is needed to compare subjects with elderly generation. Fourth, the make test for iliopsoas strength in subjects with LES cannot be generalized to the other muscles and positions. Further study is needed to investigate test-retest reliability for other muscles in various positions of make test.

\section{CONCLUSIONS}

The strength measurement of iliopsoas muscle in make test can be more reliable than break test in subjects with LES in supine position. The make test for iliopsoas muscle in supine position can be recommended in subjects with LES

\section{Key Points}

Question Can the strength measurement of iliopsoas muscle be reliable in subjects with lumbar extension syndrome in supine position?

Findings The findings of this study reported that the strength measurement of iliopsoas muscle in make test in supine position can be more reliable than break test in subjects with lumbar extension syndrome.

Meaning The intervention for strength measurement of iliopsoas muscle should be different especially in subjects with lumbar extension syndrome in supine position.

\section{Article information}

Conflict of Interest Disclosures: None.

Funding/Support: This work was supported by the National Research Foundation of Korea (NRF) grant funded by the Korea government (MSIT) (No. 2017R1C1B5076 172).

Acknowledgment: None.

Ethic Approval: IRB was approved (No. 1041849-201603BM-015-01).

\section{REFERENCES}

1. Sahrmann SA. Diagnosis and treatment of movement impairment syndromes. St Louis: Mosby; 2002.

2. Comerford M, Mottram S. Kinetic control: the management of uncontrolled movement. Australia: Churchill Livingstone; 2012. 
3. Scott DA, Bond EQ, Sisto SA, et al. The intra- and interrater reliability of hip muscle strength assessments using a hand held versus a portable dynamometer anchoring station. Arch Phys Med Rehabil. 2004;85(4): 598-603.

4. Norris CM. Spinal stabilization: 4. Muscle imbalance and the low back. Physiotherapy. 1995;81(3):127-138.

5. Wadsworth CT, Krishnan R, Sear M, et al. Intrarater reliability of manual muscle testing and hand-held dynametric muscle testing. Phys Ther. 1987;67(9):13421347.

6. Nadler SF, Malanga GA, DePrince M, et al. The relationship between lower extremity injury, low back pain, and hip muscle strength in male and female collegiate athletes. Clin J Sport Med. 2000;10(2):89-97.

7. Schwartz S, Cohen ME, Herbison GJ, et al. Relationship between two measures of upper extremity strength: manual muscle test compared to hand-held myometry. Arch Phys Med Rehabil. 1992;73(11):1063-1068.

8. Agre JC, Magness JL, Hull SZ, et al. Strength testing with a portable dynamometer: reliability for upper and lower extremities. Arch Phys Med Rehabil 1987;68(7): 454-458.

9. Arnold CM, Warkentin KD, Chilibeck LES. The reliability and validity of handheld dynamometry for the measurement of lower-extremity muscle strength in older adults. J Strength Cond Res. 2010;24(3):815-824.
10. Norton BJ, Sahrmann SA, Van Dillen FL. Differences in measurements of lumbar curvature related to gender and low back pain. J Orthop Sports Phys Ther. 2004; 34(9):524-534.

11. Kendall KD, Schmidt C, Ferber R. The relationship between hip-abductor strength and the magnitude of pelvic drop in patients with low back pain. $J$ Sport Rehabil. 2010;19(4):422-435.

12. Markhede G, Gunnar G. Measurement of strength of hip joint muscles. Scand J Rehabil Med. 1980;12(4):169174.

13. Koh, EK, Jung DY. The reliability of flexor hallucis longus stretch test in subjects with asymmetric hallux valgus angles. J Kor Phys Ther. 2016;28(2):124-127.

14. Stratford PW, Balsor BE. A comparison of make and break tests using a hand-held dynamometer and the KinCom. J Orthop Sports Phys Ther. 1994;19(1):28-32.

15. McBeth JM, Earl-Boehm JE, Cobb SC, et al. Hip muscle activity during 3 side-lying hip-strengthening exercises in distance runners. J Athl Train. 2012;47(1): 15-23.

16. Desmyttere G, Gaudet S, Begon M. Test-retest reliability of a hip strength assessment system in varsity soccer players. Physical Therapy in Sport. 2019;37(2): 138-143. 